\title{
A Novel Ensemble Method for Predicting Wheat Yield Using Feature Selection-based Deep Learning and Hyperspectral Vegetation Indices
}

\section{Shuaipeng Fei}

Institute of Farmland Irrigation, Chinese Academy of Agricultural Sciences

Lei Li

National Wheat Improvement Centre, Institute of Crop Sciences, Chinese Academy of Agricultural

Sciences

Zhiguo Han

PhenoTrait Laboratory, PhenoTrait Technology Co. Ltd

Zhen Chen ( $\nabla$ chenzhen@caas.cn )

Institute of Farmland Irrigation, Chinese Academy of Agricultural Sciences

\section{Yonggui Xiao}

National Wheat Improvement Centre, Institute of Crop Sciences, Chinese Academy of Agricultural Sciences

\section{Research Article}

Keywords: wheat yield, hyperspectral, vegetation index, deep neural network, feature selection

Posted Date: February 28th, 2022

DOl: https://doi.org/10.21203/rs.3.rs-1392054/v1

License: (c) (1) This work is licensed under a Creative Commons Attribution 4.0 International License. Read Full License 
1 A Novel Ensemble Method for Predicting Wheat Yield Using Feature

2 Selection-based Deep Learning and Hyperspectral Vegetation Indices

3 Shuaipeng $\mathrm{Fei}^{1}$, Lei $\mathrm{Li}^{2}$, Zhiguo $\mathrm{Han}^{3}$, Zhen Chen ${ }^{1 *}$, Yonggui Xiao ${ }^{2 *}$

41 Institute of Farmland Irrigation, Chinese Academy of Agricultural

5 Sciences/ Key Lab of Efficient Irrigation Engineering, Ministry of

6 Agriculture \& Rural Affairs/ Key Laboratory of Water-Saving Agriculture

7 of Henan Province, Xinxiang 453002, China

82 National Wheat Improvement Centre, Institute of Crop Sciences,

9 Chinese Academy of Agricultural Sciences, Beijing 100081, China

103 PhenoTrait Laboratory, PhenoTrait Technology Co. Ltd, Beijing 100096,

China

*Correspondence: Zhen Chen, Email: chenzhen@caas.cn and Yonggui Xiao, Email: xiaoyonggui@caas.cn

\section{Abstract}

Background: Wheat is an important food crop globally, and timely prediction of wheat yield in breeding efforts can improve the efficiency of selection. Traditional plant breeding based on grain yield selection is time-consuming, costly, and destructive. There is a great need for innovative methods to enhance efficiency and accelerate genetic gains in the breeding cycle.

Results: In this study, a new ensemble learning method was developed to predict wheat yield by combining hyperspectral data and deep learning-based regression technology. For this, 207 wheat cultivars and breeding lines were grown under full and limited irrigation treatments, and their canopy hyperspectral reflectance was measured at the flowering, early grain fill (EGF), mid grain fill (MGF), and late grain fill (LGF). Firstly 115 vegetation indices (VIs) were extracted from the hyperspectral 
reflectance and combined with four feature selection methods i.e., mean decrease impurity (MDI), Boruta, FeaLect, and Relief to train deep neural network (DNN) models for yield prediction. Then, a novel ensemble learning framework was developed by combining the predicted values of selected and the full features using multiple linear regression (MLR). The results show that feature selection methods achieved higher yield prediction accuracy than the full features, where MDI method performed best across growth stages, with the mean $R^{2}$ ranging from $0.634-0.666$ (mean RMSE $=0.926-0.967 \mathrm{t} \mathrm{ha}^{-1}$ ). The proposed ensemble method outperformed all the FS methods across growth stages, with the mean $R^{2}$ ranging from 0.648-0.679 (mean RMSE $\left.=0.911-0.950 \mathrm{t} \mathrm{ha}^{-1}\right)$.

Conclusions: By integrating multiple FS methods and DNN, more prediction potential of hyperspectral data can be exploited, and this ensemble method is also applicable to trait estimation of other crops.

Keywords: wheat yield; hyperspectral; vegetation index; deep neural network; feature selection

\section{Background}

Declining crop yields due to climate change and global population growth are putting the global food supply at risk $[1,2]$. The development of a large number of superior resistant varieties through plant breeding efforts is an immediate solution. In most cases, improving yields is the primary goal of crop breeding programs [3]. However, yield is influenced by both quantitative and qualitative traits, and measuring yield in large breeding populations consisting of thousands of genotypes can be time-consuming and laborious [3-5]. Secondary traits can help breeders predict grain yield at early stages to reduce the time and cost [6], while the traditional manual-based trait survey methods are still inefficient. In 
recent years, developments in remote sensing and spectroscopy sensor technologies have helped to establish low-cost, high-throughput phenotyping platforms (HTPP) that can collect large amounts of data related to yield at different stages under various growth environments in breeding efforts $[4,6-8]$.

The spectroscopy science of agriculture is based on the ability to measure different wavelengths of electromagnetic energy interacting with different parts of a growing plant [8]. The goal of spectral science is to quantify phenotypes through interactions between light and plants, such as reflected, absorbed, transmitted, and/or emitted photons [8]. Commonly used sensors in precision agriculture are hyperspectral, RGB, multispectral, and thermal [9-12]. Compared to other sensors, hyperspectral sensors with a large number of continuous bands have been successfully applied to estimate crop parameters including yield, biomass, leaf area index (LAI), and chlorophyll content [13-15]. In addition to the raw bands, hyperspectral data can also be derived from integer and fractional order derivatives to reveal more hidden information related to crop growth $[15,16]$. The reflectance of electromagnetic energy at different wavelengths is usually summarized as the vegetation index [8], which is further used to predict physiological problems or agronomic traits of plants. Related studies combined a large number of vegetation indices in various wavelength regions to assess crop parameters $[15,17]$, and different VIs can complement each other to provide more information related to plant growth.

Parsing large data sets acquired by high-throughput phenotyping platforms requires intensive computational and statistical analysis, which remains a challenge in plant breeding programs [18]. Nowadays, many 
statistical and machine learning-based regression techniques, such as support vector regression (SVR), random forest regression (RFR) and extreme learning machines (ELM), have been applied to build predictive models for plant traits and achieve accurate predictions [15,19-21]. As a subfield of machine learning, deep learning can automatically learn representations from data using multi-layer architecture [22]. The architecture supports complex nonlinear functions, which are learned from the hierarchical output of the previous layers [22]. Deep learning methods such as convolutional neural network $(\mathrm{CNN})$, deep neural network (DNN), and residual neural network (ResNet) have yielded higher accuracy in various regression and classification tasks in the field of precision agriculture [23-25].

To obtain accurate yield predictions and avoid model overfitting, machine learning algorithms benefit from using feature selection (FS) methods to reduce the redundancy of the data [26]. FS methods such as recursive feature elimination (RFE), Pearson correlation coefficient (PCC), random forest-based mean decrease impurity (MDI) and Partial least squares based variable importance in the projection (VIP) methods have been used in estimating crop parameters ranging from alfalfa and soybean yield prediction to sorghum leaf chlorophyll concentration estimation [3, 16, 17]. Each FS method has its unique focus, and most studies have utilized only an individual FS method for modeling, which inspired us to combine the characteristics of multiple FS methods. Ensemble learning such as stacking regression is gaining a lot of attention in the machine learning community. Ensemble models usually achieve better prediction results than individual models [17, 27, 28]. During the process of ensemble learning, a set of basic individual learners is first 
used for training, and then the predictions of all learners are combined to make the final prediction. In general, heterogeneity among basic learners motivates the accuracy-improving effect of ensemble models [29]. Similarly, there are differences in the features selected by various FS methods, resulting in heterogeneity among the output predictions, and combining multiple FS methods in a stacking pattern has the potential to obtain higher prediction accuracy than individual FS methods and full features.

Based on the above descriptions, the main objectives of this study are: (1) to explore the potential application of hyperspectral data and DNN in predicting wheat yield, (2) to compare the yield prediction accuracy of individual feature selection methods and proposed ensemble method, and (3) to identify the optimal stage for acquiring hyperspectral data at late wheat growth.

\section{Materials and Methods}

\section{Experimental design}
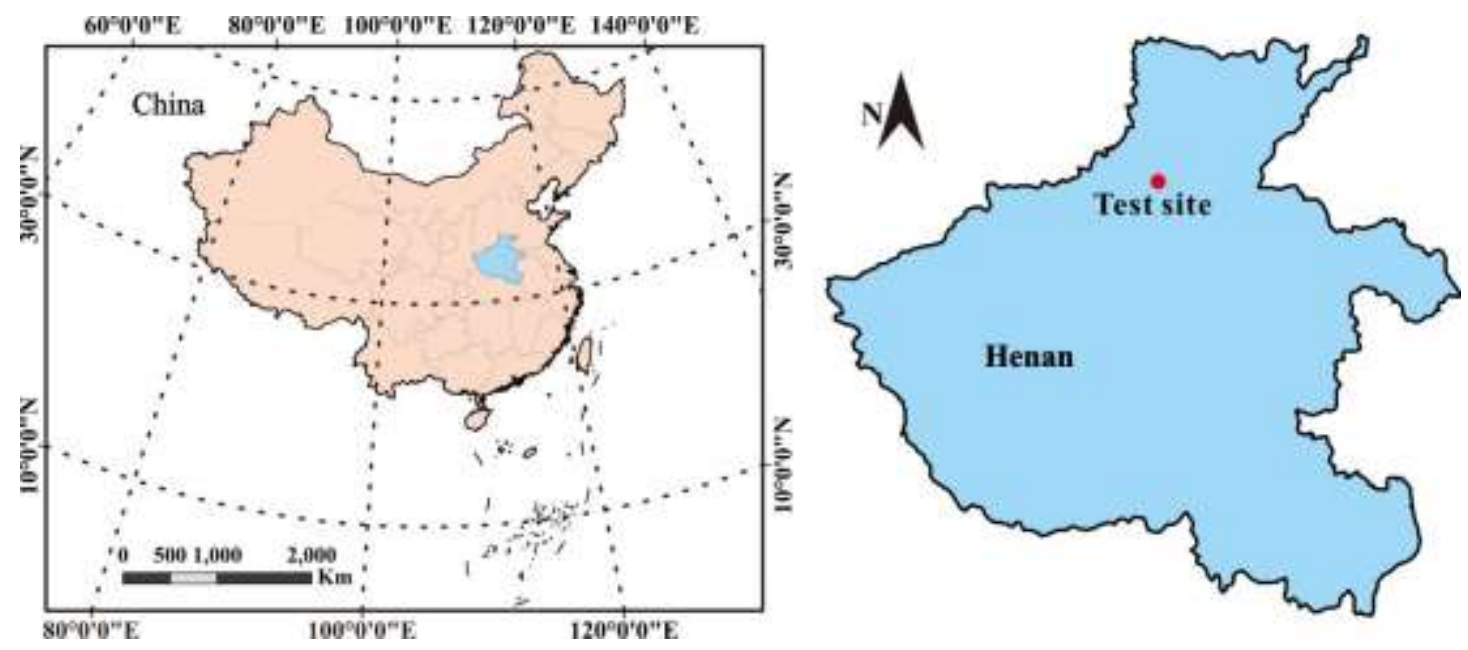

Fig. 1 Test site location.

This study adopted a diverse panel of 207 varieties. During growing season 2018-2019, all accessions were cultivated at the research station of 
129 Chinese Academy of Agricultural Sciences (CAAS) at Xinxiang $\left(35^{\circ} 18^{\prime} \mathrm{N}\right.$, $130113^{\circ} 51^{\prime} \mathrm{E}$; Henan Province, China) (Fig. 1) under two water irrigation 131 levels, full and limited irrigation. The field experiments were set up in 132 randomized complete blocks with two replications. Each plot was $4.2 \mathrm{~m}^{2}$ 133 in size, with dimensions of $3 \mathrm{~m} \times 1.4 \mathrm{~m}$ and six rows with $0.2 \mathrm{~m}$ spacing. 134 At the tillering stage, both the full and limited irrigation treatments 135 received $250 \mathrm{~mm}$ of water, with irrigation continuing for the full 136 irrigation treatment at the early jointing, heading, and early grain filling 137 stages. The application of fertilizer was optimized based on the soil 138 conditions in the area. All plants were harvested at physiological maturity 139 using the combined harvester. Grain yield was measured at a $12.5 \%$ grain 140 moisture level. The overall flow of this study is shown in Fig. 2. 


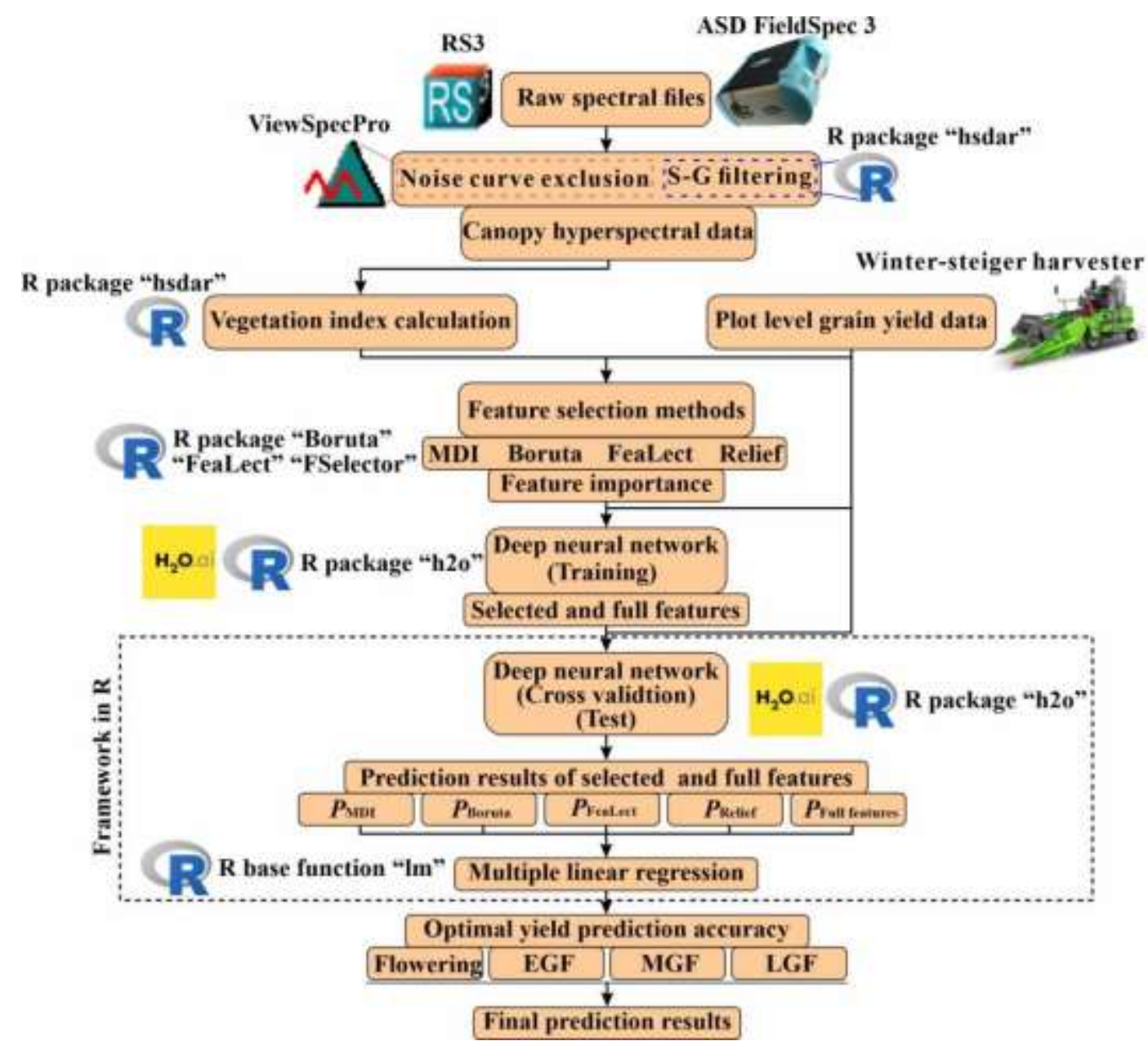

Fig. 2 Overall workflow conducted in this study.

MDI, mean decrease impurity; EGF, early grain fill; MGF, min grain fill;

LGF, late grain fill; $P$, model predictions.

\section{Hyperspectral data acquisition and processing}

A high-spectral-resolution spectrometer (Fieldspec 3, Analytical Spectral Devices ASD, Boulder, CO, USA) connected with a $25^{\circ}$ field of view fiber optic was used to collect the canopy reflectance parameters of each plot from 400 to $2500 \mathrm{~nm}$. The visible-to-near-infrared range (350-1000nm) had a spectral resolution of $3 \mathrm{~nm}$, while the shortwave

152 infrared region had a spectral resolution of $10 \mathrm{~nm}(1000-2500 \mathrm{~nm})$. The sensor was placed $100 \mathrm{~cm}$ above the canopy in a nadir position and operated vertically. Canopy reflectance was measured at four separate 
sites in each plot between 11:00 a.m. and 1:00 p.m. local time on a clear day. For each site, ten readings were taken, and the average of these 40 readings was used to calculate the canopy reflectance of the plot. Before measuring canopy reflectance, a $\mathrm{BaSO} 4$ calibration panel was used to estimate incoming radiation and reflectance. This process was done every ten plots. Spectral measurements were carried out at flowering, early grain fill (EGF), mid grain fill (MGF), and late grain fill (LGF). View Spec software (ASD Inc, Boulder, CO, USA) was used to eliminate noise from spectral curves, calculate the average of numerous spectral curves, and generate a reflectance file. To overcome the noise probability during the spectrum collecting process, the adaptive degree polynomial filter (ADPF) was used. ADPF adds a statistical heuristic to the Savitzky-Golay method to improve signal fidelity while reducing statistical noise. Following filtering, a database of 115 vegetation indices (Table S1) was created as input features to the yield prediction model.

\section{Deep neural network}

The deep neural network (DNN) has been increasingly applied to solve regression problems in various fields of research [23, 30-31]. The schematic diagram of DNN is shown in Fig. 3. DNN generally consists of an input layer, several hidden layers, and an output layer [32]. The number of features corresponds to the neurons (units) in the input layer. The hidden layer neurons perform nonlinear transformations of the original input attributes. The number of output neurons depends on the number of response variables that are predicted for continuous response variables (grain yield in this study), and it receives the output of the hidden neurons as input and produces the associated predicted values as output. 


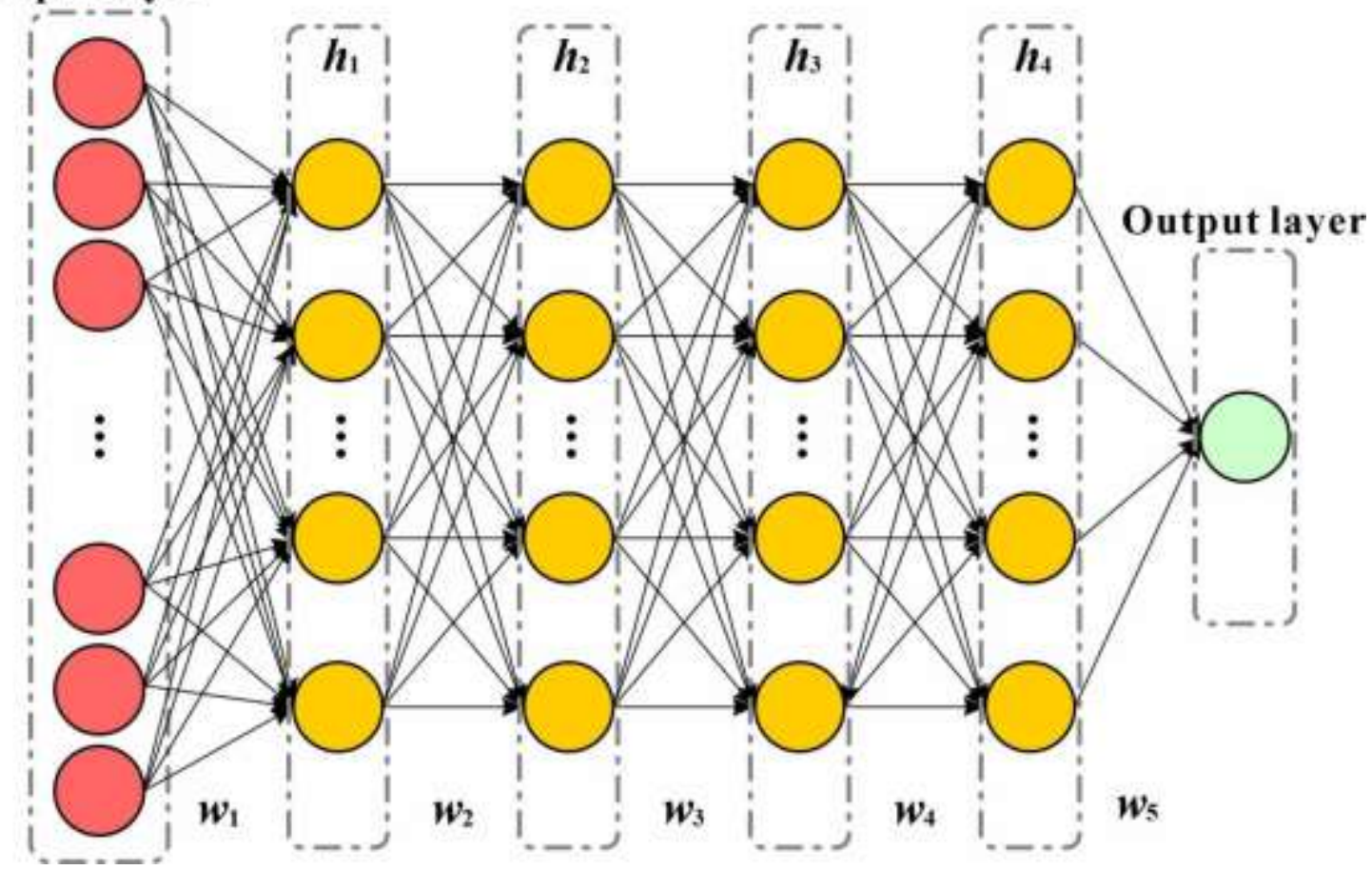

Fig. 3 A schematic diagram of the deep neural work.

$w$, weight; $h$, hidden layer.

Screening for appropriate hyperparameters is a key step in the successful implementation of deep learning models. The grid search method was applied to tune the hyperparameters. Through the literature [32], we set the following parameters: The units values used for the grid search were 50 to 150 in increments of 10, and for the hidden layers we used 3, 4, 5 and 6. The regularization method "dropout" was used to randomly remove a subgroup of hidden neurons and their connections. The activation function applied was the rectified linear activation unit (RELU) function. All DNN models were built in $\mathrm{R}$ software with the "h2o" package.

\section{Feature selection method}

\section{Mean Decrease Impurity (MDI)}

MDI is a random forest-based FS method. The random forest utilizes randomized decision trees and impurity measurements to calculate the 
importance of various features [33]. When the random forest employs the Gini index as its impurity measurement, one such technique is known as mean decrease impurity (MDI). Breiman (2001) presented to estimate the importance of a variable $k$ for predicting $y$ (i.e., grain yield) by cumulate the weighted impurity decreases $\left(p(t) \Delta i\left(s_{t}, t\right)\right)$ for all nodes $t$ where $k$ is used and averaged over all $N_{T}$ trees in the forest as in the following equation:

$$
M D I_{k}=\frac{1}{N_{T}} \sum_{T} \sum_{t \in T: v\left(s_{t}\right)=k} p(t) \Delta i\left(s_{t}, t\right)
$$

where $p(t)$ represents the proportion $N t / N$ of sample reaching $t$, and $v\left(s_{t}\right)$ represents the variable used in split $s_{t}$.

\section{Boruta}

Boruta is a wrapper method developed based on the random forest algorithm. The principle of Boruta is different with MDI method. The Boruta algorithm is an extension of the idea proposed by [34]. The significant input parameters are defined by comparing the correlation between the real features and the random probes. Boruta calculates the Z-scores for each input predictor concerning the shading attribute [35]. The significant factors of the predictors are determined by the distribution of the Z-score indicators. Based on the significant factors determined by Boruta, a minimum optimal FS strategy was used, by ranking and residuals, followed by stepwise modeling. The outlined Boruta algorithm is as follows [11]: (a) The eigenvalues of each feature matrix consisting of the independent and dependent variables are shuffled to obtain shadow features, and then the true and shadow features are combined to form a new feature matrix for training. (b) Inputting the newly constructed feature matrix to obtain the trained feature importance model. (c) 
Calculate the scores of the real and shadow features and determine the maximum Z-score of the shadow features. (d) The real features with scores greater than the maximum Z-score were classified as important factors, while the real features with scores significantly less than the maximum Z-score were classified as unimportant factors. Repeat steps (a)-(d) until the importance of all feature attributes is marked as "important" or "not important". In this study, the importance ranking was based on the average Z-score score of each feature.

\section{FeaLect}

FeaLect is a FS method proposed by [36] based on combinatorial analysis of LASSO. Set $B$ be a random sample of size $m$, generated by selecting without replacement from the given training data $D$, where $n=$ $|D|$ and $\gamma \in(0,1)$ represents a parameter that controls the size of sample set. The Lars algorithm is applied to efficiently recover the entire regularization path using training set $\mathrm{B}$. Let $F_{k}^{B}$ be the set of selected features by the Lasso when $\lambda$ allowing the selection of $k$ features. The number of selected features is decreasing in $\lambda$ and we get the formula:

$$
\emptyset=F_{0}^{B} \subset \cdots F_{k}^{B} \subset F_{k+1}^{B} \subset \cdots \subset F_{d}^{B}=F
$$

For each feature $f$, a scoring mechanism was defined based on whether or not it is selected in $F_{k}^{B}$ :

$$
S_{k}^{B}(f):=\left\{\begin{array}{l}
\frac{1}{k} \text { if } f \in F_{k}^{B} \\
0 \text { otherwise }
\end{array}\right.
$$

The above randomized process is randomly cycled several times for various random subsets $B$ to compute the average score of $f$ when $k$ features are selected. The sum of average scores is then used to calculate the total score for each feature:

$$
S(f):=\sum_{k} \mathbb{E}_{B}\left[S_{k}^{B}(f)\right]
$$




\section{Relief}

The Relief algorithm's central principle is to evaluate the quality of attributes with weights greater than the thresholds by comparing the value of an attribute between a given instance and the two nearest instances [37]. For the regression problem, a detailed procedure has been given [38]. The Relief algorithm is given by the following equation:

$$
\Delta W(f)=-\frac{\operatorname{diff}(f, R, H)}{m}+\frac{\operatorname{diff}(f, R, M)}{m}
$$

Where $f$ and $W$ represent the feature and weight, respectively. $m$ and $R$ represent the number of training data and target data, respectively. $H$ and $M$ represent the nearest neighbor of the target with same and opposite class, respectively.

\section{Framework of ensemble feature selection}

The proposed ensemble method based on DNN in this study draws on the verification pattern of the stacking ensemble method (Fig. 4). According to the importance ranking of each FS method, each feature is input to the DNN in turn until the training accuracy reaches the maximum, at which time the input features are considered the optimal combination of features, together with the full features, five deep neural network models are generated. The main steps for ensemble models applied in this study are as follows [39]:

(a) The original datasets of each FS method and full features are divided into a training dataset and test dataset in the ratio of $4: 1$;

(b) For each DNN model at level-1, the 5 fold cross-validation is used to train and output the val_pre dataset for the validation dataset and test_pre set for the test dataset in each fold. The val_pre sets are combined as the new training dataset and the test pre sets are averaged as the new testset; 
(c) The new training set is employed to train the ensemble feature 279 selection by multiple linear regression (MLR) at level-2 through 5 fold 280 cross-validation and to output the final prediction of new test dataset for 281 each fold, and then averaged over them to obtain final predictions.

To avoid interference by chance, the above process was repeated 20 times, the average accuracy parameters of 100 tests generated in the cross-validation process are used to evaluate model performance.

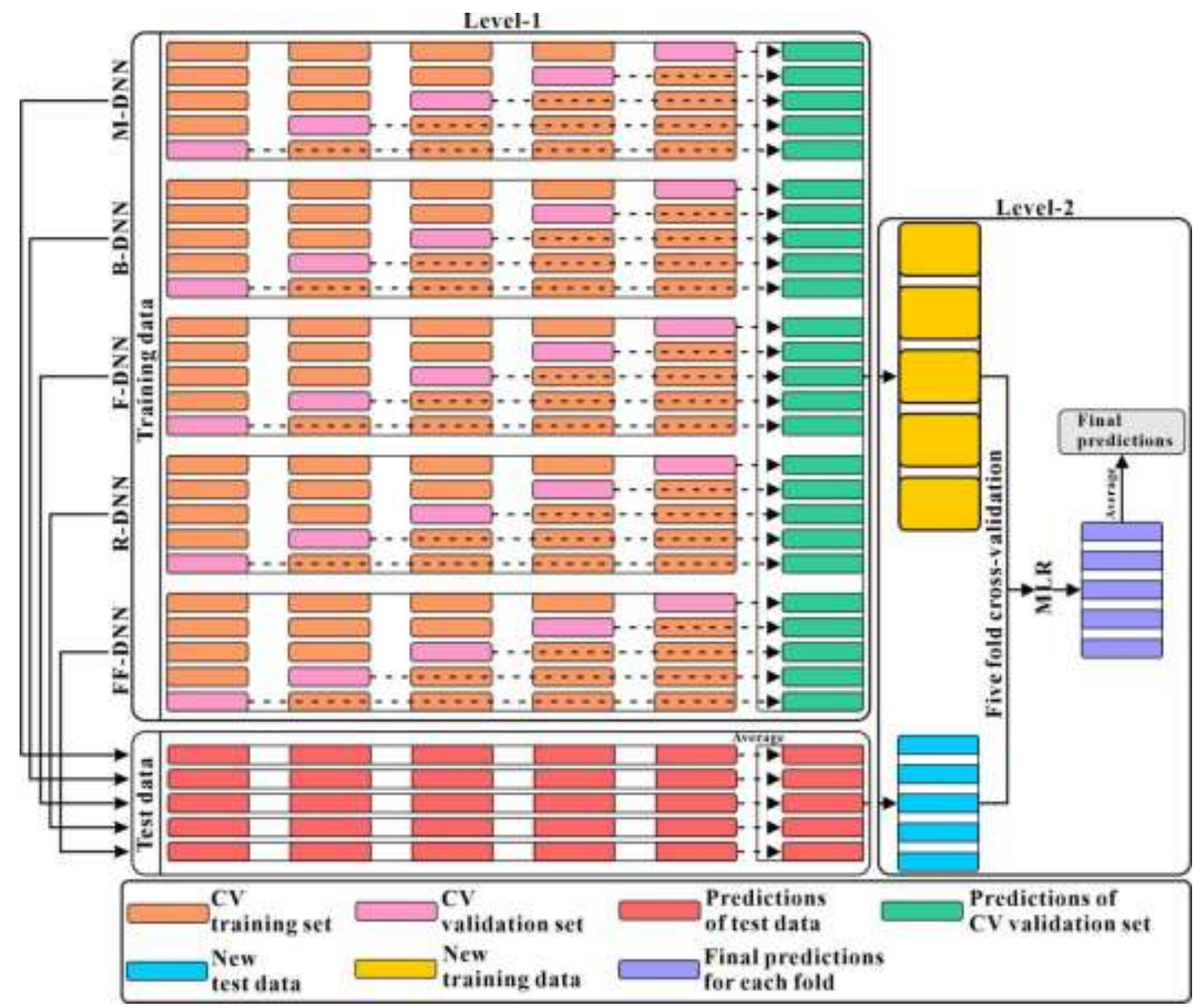

Fig. 4 Structure of the ensemble learning model.

DNN, deep neural network; M, mean decrease impurity; B, Boruta; F,

FeaLect; R, Relief; FF, full features; CV, cross-validation; MLR, multiple linear regression. 
Coefficient of determination $\left(R^{2}\right)$ and root mean square error (RMSE) were used to evaluate the accuracy of the yield prediction model. The equations are as follow:

$$
\begin{gathered}
R^{2}=1-\frac{\sum_{i=1}^{n}\left(\hat{y}_{i}-y_{i}\right)^{2}}{\sum_{i=1}^{n}\left(y_{i}-\bar{y}\right)^{2}} \\
\text { RMSE }=\sqrt{\frac{\sum_{i=1}^{n}\left(\hat{y}_{l}-y_{i}\right)^{2}}{n}}
\end{gathered}
$$

where $y_{i}$ and $\hat{y}_{i}$ are the measured and the predicted grain yield, respectively. $\bar{y}$ is the mean of measured grain yield, and $n$ is the total number of testing samples. A larger $R^{2}$ and a smaller RMSE indicates a stronger predictive capability of the model.

\section{Statistical analysis}

A mixed linear model was adopted to test the significance of variation between genotypes, irrigation treatments, and their interactions for measured and predicted grain yield. The equation of the model is as follows [40]:

where $Y$ is the response demonstrated by fixed effect $(\beta)$ and random effect $(\mu)$ with random error $(\varepsilon) . X$ and $Z$ denote fixed and random effects, respectively. Broad-sense heritability refers to the percentage of genetic variation to the total variation in the phenotype, with a value between 0 and 1 . Heritabilities of 0 and 1 indicate that phenotypic variation is entirely influenced by environmental and genetic factors, respectively. Heritability was calculated by the following formula:

$$
H^{2}=\sigma_{\mathrm{g}}^{2} /\left(\sigma_{\mathrm{g}}^{2}+\sigma_{\varepsilon}^{2} / r\right)
$$


where $r$ represents the number of replications per treatment; $\sigma_{\mathrm{g}}{ }^{2}$ and $\sigma_{\varepsilon}{ }^{2}$ are the genotypic and error variances, respectively.

\section{Results}

\section{Descriptive statistics of measured wheat yields}

The descriptive statistics and distribution of measured yields from both irrigation treatments are shown in Fig. 5. The resulting mean yield values for the full and limited irrigation treatments were 9.64 and $7.79 \mathrm{t}$ $\mathrm{ha}^{-1}$, respectively. The yield under full irrigation treatment had a wider range of distribution with a higher standard deviation (1.43 $\left.\mathrm{t} \mathrm{ha}^{-1}\right)$ compared to limited irrigation treatment $\left(1.11 \mathrm{t} \mathrm{ha}^{-1}\right)$. The results of the Shapiro-Wilk test $(P<0.05)$ indicated that the yields under both irrigation treatments were normally distributed.

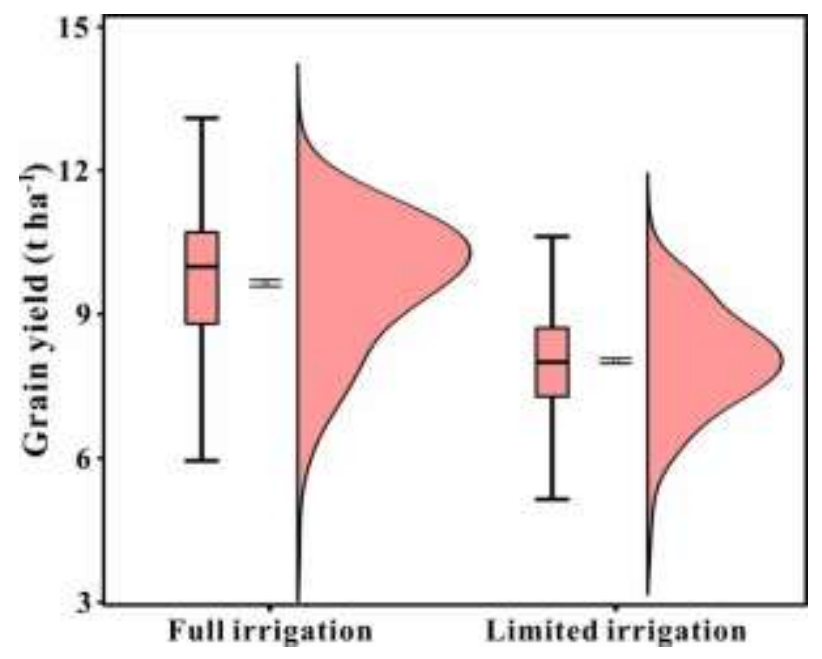

Fig. 5 Distribution of wheat yield in full and limited irrigation treatments.

\section{Feature selection results}

The 115 VIs were ranked using the MDI, Boruta, FeaLect, and Relief strategies, respectively. The detailed ranking of VIs is shown in Table S2-S5. The results show that there are differences in the ranking of VIs among the four FS methods. Meanwhile, the performance of some VIs was stable and excellent. For example, the Datt8, PWI, Datt7, DPI, 
PRI, PRI_norm, mREIP, and REP_Li were ranked in the top 30 for all 337 four FS methods at flowering (Table S2-S5). These better-performing 338 indices form the basis for the model's outperformance.
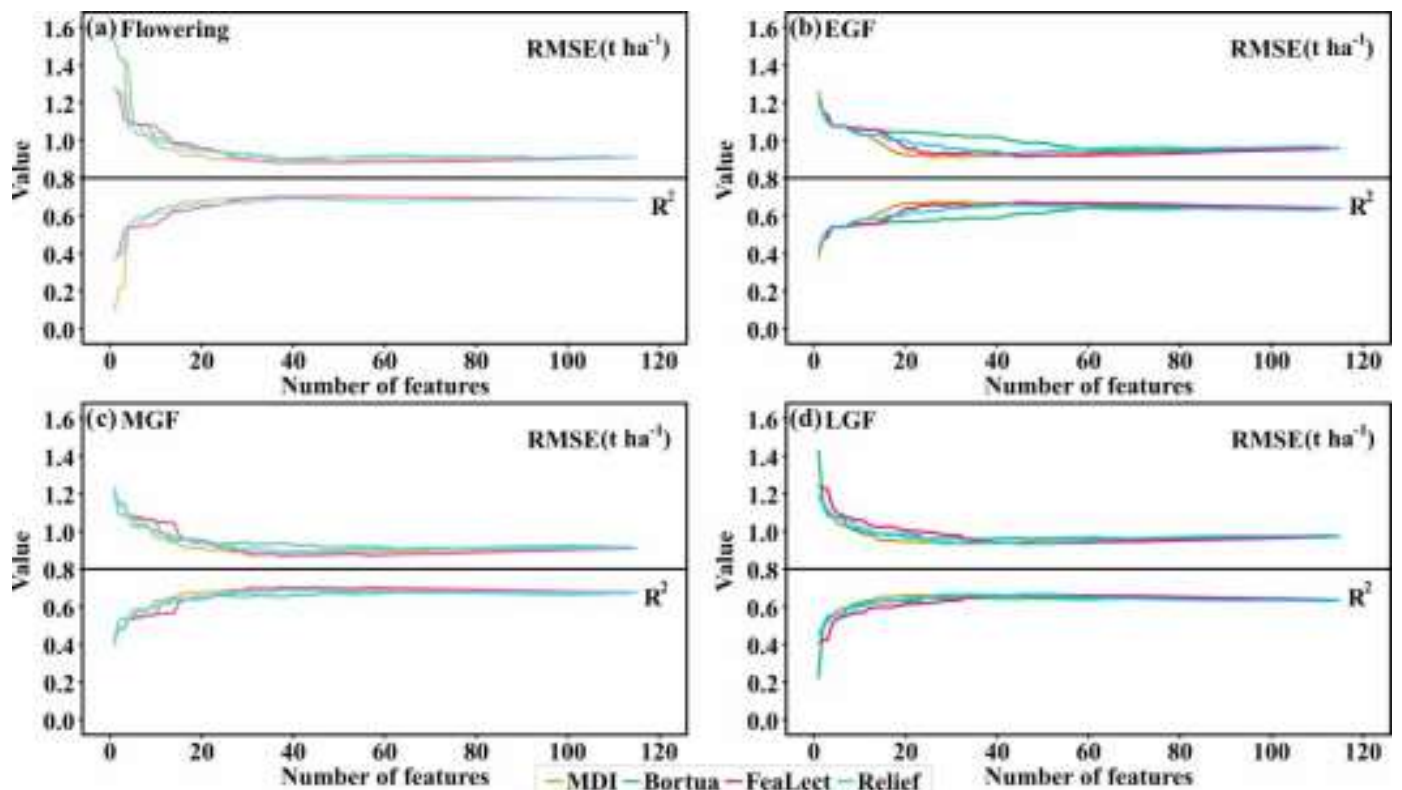

Fig. 6 Deep neural network model training accuracy as a function of the number of features.

342 MDI, mean decrease impurity; EGF, early grain fill; MGF, min grain fill; 343 LGF, late grain fill.

Vegetation indices were iteratively added into the DNN model according to the ranking and updated the model training performance $\left(R^{2}\right.$ and RMSE) until all 115 indices were included to further investigate the

347 features with superior performance. With the input of more features, the 348 training accuracy of DNN model first rose to the best and then slightly 349 decreased (Fig. 6). The training accuracy of the MDI method was the 350 highest at all measured stages. Furthermore, the MDI method can achieve 351 the best prediction accuracy with the minimum number of features at 352 early, mid, and late grain filling stages. The performances of the 353 remaining FS methods were variable across the stages. For each FS 354 method, the number of features that achieve the best training accuracy 
was selected for the ensemble FS model development. The Venn diagrams (Fig. 7) are used to represent the number of features that are common and unique to multiple FS methods.

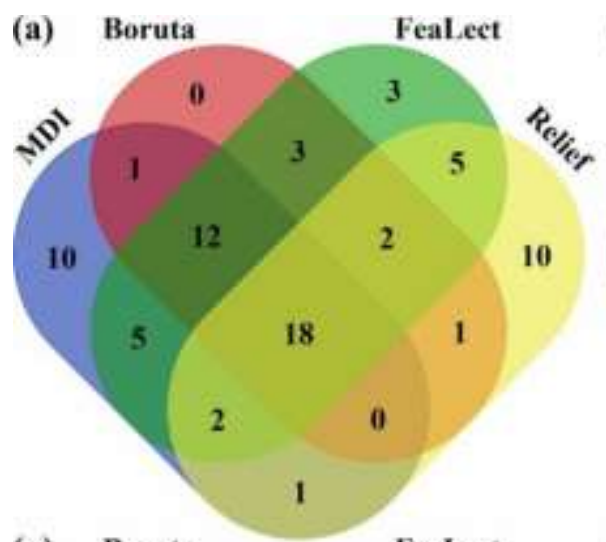

(c) Boruta Fealect

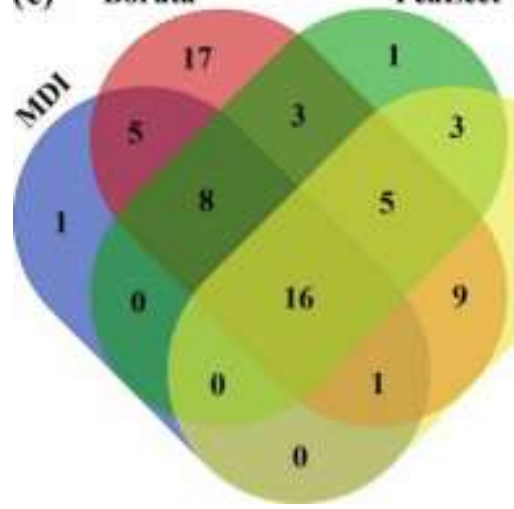

(b) Boruta
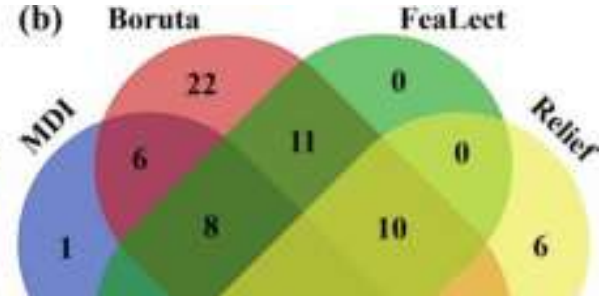

169 1 0

(d) Beruta Fealect

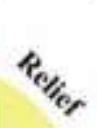

8

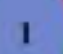

13

17

1

0

0

Fig. 7 The Venn diagram of selected features for each feature selection method. (a) flowering, (b) early grain fill, (c) mid grain fill, and (d) late grain fill.

\section{Performance of yield prediction model}

To validate the model adaptability, the DNN prediction accuracy of selected features, all features and ensemble method on the test set was analyzed, the accuracy statistics are shown in Fig. 8. For the FS methods of flowering, the MDI method yielded the highest mean $R^{2}$ of 0.636 $\left(\right.$ mean $\left.\mathrm{RMSE}=0.964 \mathrm{t} \mathrm{ha}^{-1}\right)$, followed by Boruta method (mean $R^{2}=$ 0.627, mean RMSE $\left.=0.977 \mathrm{tha}^{-1}\right)$ and FeaLect method (mean $R^{2}=0.617$, mean RMSE $=0.990 \mathrm{t} \mathrm{ha}^{-1}$ ). Compared to full features (mean $R^{2}=0.604$, mean $\operatorname{RMSE}=1.006 \mathrm{t} \mathrm{ha}^{-1}$ ), prediction accuracy of Relief was slightly 
lower (mean $R^{2}=0.589$, mean $\mathrm{RMSE}=1.030 \mathrm{t} \mathrm{ha}^{-1}$ ). The best predictive performance of EGF was achieved by the MDI method with the mean $R^{2}$ value of 0.634 and a RMSE value of $0.967 \mathrm{t} \mathrm{ha}^{-1}$, followed by the Boruta method (mean $R^{2}=0.612$, mean $\operatorname{RMSE}=0.995 \mathrm{t} \mathrm{ha}^{-1}$ ). It was also noted that the FeaLect method (mean $R^{2}=0.608$, mean RMSE $=1.001 \mathrm{t} \mathrm{ha}^{-1}$ ) had a similar predictive result to the Relief method (mean $R^{2}=0.607$, mean $\operatorname{RMSE}=1.003 \mathrm{t} \mathrm{ha}^{-1}$ ). Full features yielded the lowest mean $R^{2}$ of 0.570 (mean RMSE $=1.046 \mathrm{tha}^{-1}$ ). The best FS method of MGF was also achieved by MDI (mean $R^{2}=0.666$, mean $\mathrm{RMSE}=0.926 \mathrm{t} \mathrm{ha}^{-1}$ ), followed by Boruta (mean $R^{2}=0.658$, mean RMSE $=0.938 \mathrm{tha}^{-1}$ ), Relief (mean $R^{2}$ $=0.643$, mean $\left.\mathrm{RMSE}=0.958 \mathrm{t} \mathrm{ha}^{-1}\right)$, FeaLect (mean $R^{2}=0.639$, mean $\mathrm{RMSE}=0.963 \mathrm{t} \mathrm{ha}^{-1}$ ), and full features (mean $R^{2}=0.616$, mean RMSE $=$ $\left.0.992 \mathrm{t} \mathrm{ha}^{-1}\right)$. Differ from the first three stages, the FS method with the highest prediction accuracy at LGF was Boruta (mean $R^{2}=0.643$, mean RMSE $=0.957 \mathrm{t} \mathrm{ha}^{-1}$ ), followed by MDI (mean $R^{2}=0.639$, mean RMSE $=0.962 \mathrm{t} \mathrm{ha}^{-1}$ ). The prediction performance of methods FeaLect (mean $R^{2}$ $=0.624$, mean RMSE $=0.982 \mathrm{t} \mathrm{ha}^{-1}$ ), Relief (mean $R^{2}=0.627$, mean RMSE $\left.=0.979 \mathrm{t} \mathrm{ha}^{-1}\right)$ and full features (mean $R^{2}=0.622$, mean RMSE $=$ $0.985 \mathrm{tha}^{-1}$ ) was similar. Compared to the individual FS method with the highest prediction accuracy, the ensemble method improved the mean $R^{2}$ value to $0.648,0.650,0.679$ and 0.652 for flowering, EGF, MGF and LGF, respectively, also the values of RMSE had decreased (Fig. 8). In the process of the ensemble method, MDI contributed more and the regression coefficients assigned within MLR were higher in all periods (Fig. 9). 

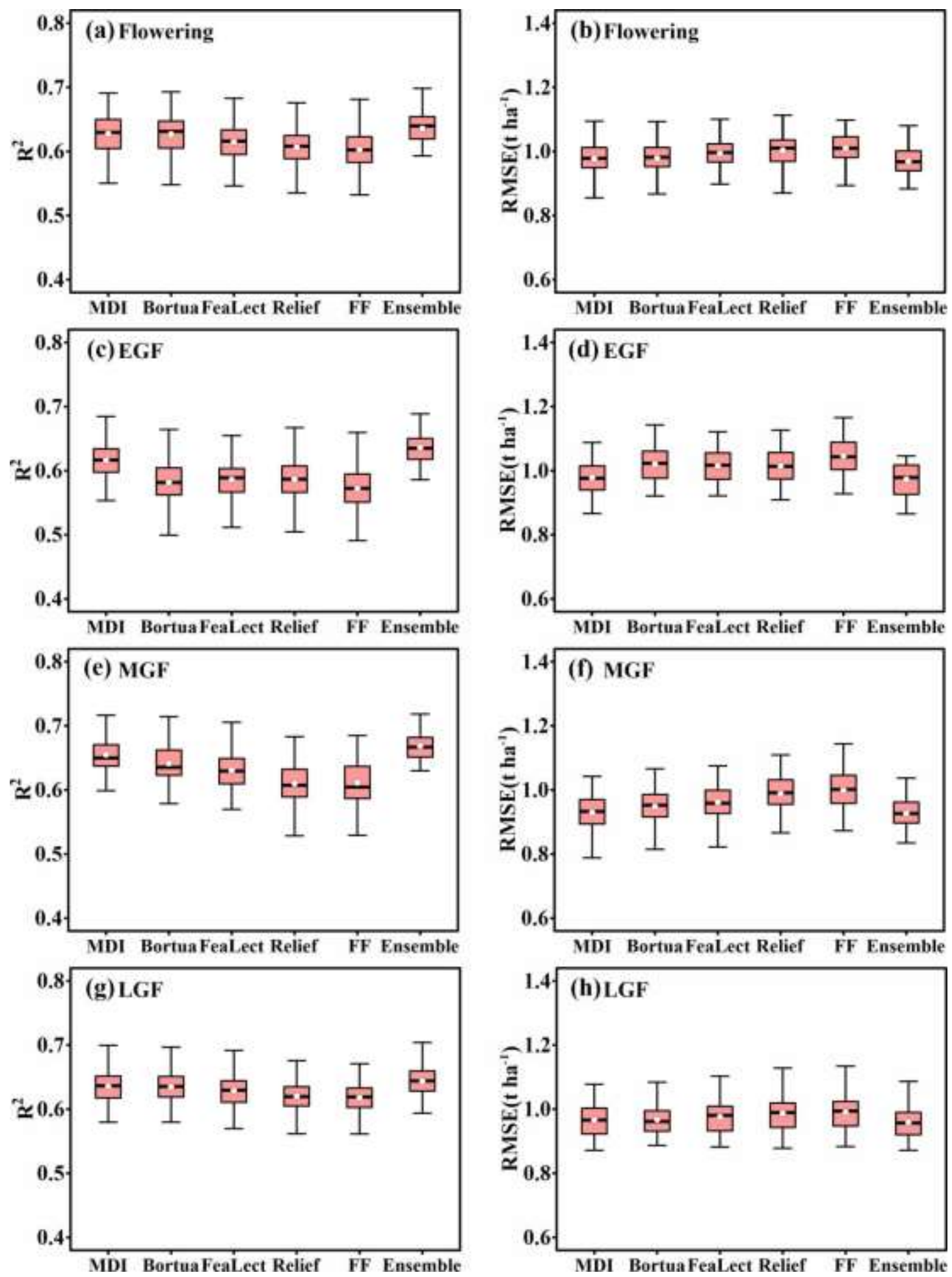

399 Fig. 8 The statistical distributions of the prediction accuracy of various

$400 \quad$ FS and ensemble methods.

401 EGF, early grain fill; MGF, min grain fill; LGF, late grain fill. 
403

404

405

406

407

408

409

410

411

412

413

414

415

416

417

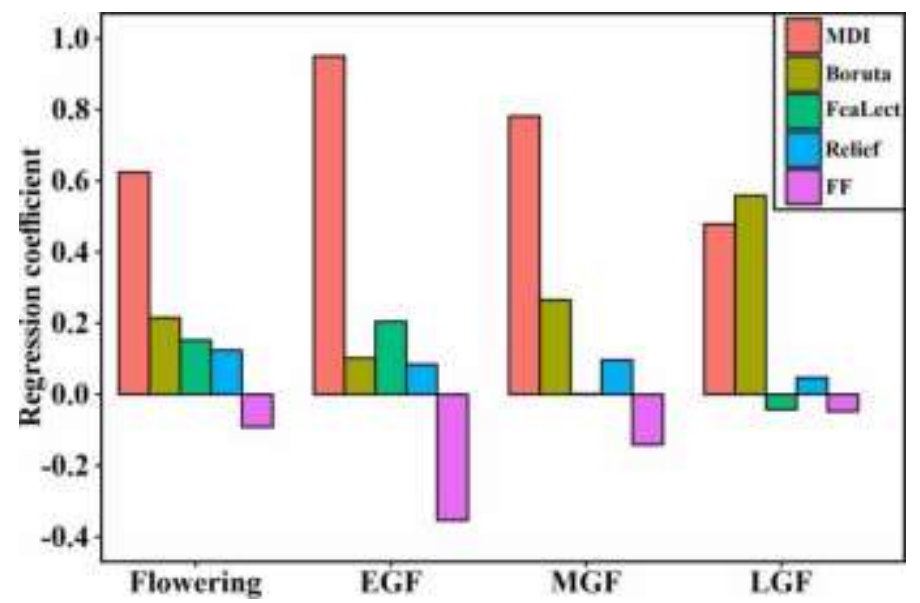

Fig. 9 Mean value of regression coefficients assigned to each feature selection method.

EGF, early grain fill; MGF, min grain fill; LGF, late grain fill.

\section{Analysis of yield prediction value}

Predicted wheat yield was output using the ensemble method for both irrigation treatments. Wheat yield under full irrigation treatment was higher than that under limited irrigation treatment for all measured stages (Fig. 10). ANOVA (Table 1) revealed that genotypes, treatments, and genotype $\times$ treatment interactions had significant effects on predicted yield for all measured stages, which was consistent with the measured yield. Similar to measured yield $\left(H^{2}=0.63\right)$, the $H^{2}$ of predicted yield was high, with the value of $0.73,0.71,0.77$, and 0.62 for flowering, EGF, MGF, and LGF across two irrigation treatments, respectively, suggesting that most of the phenotypic variation was determined by genetic factors. 


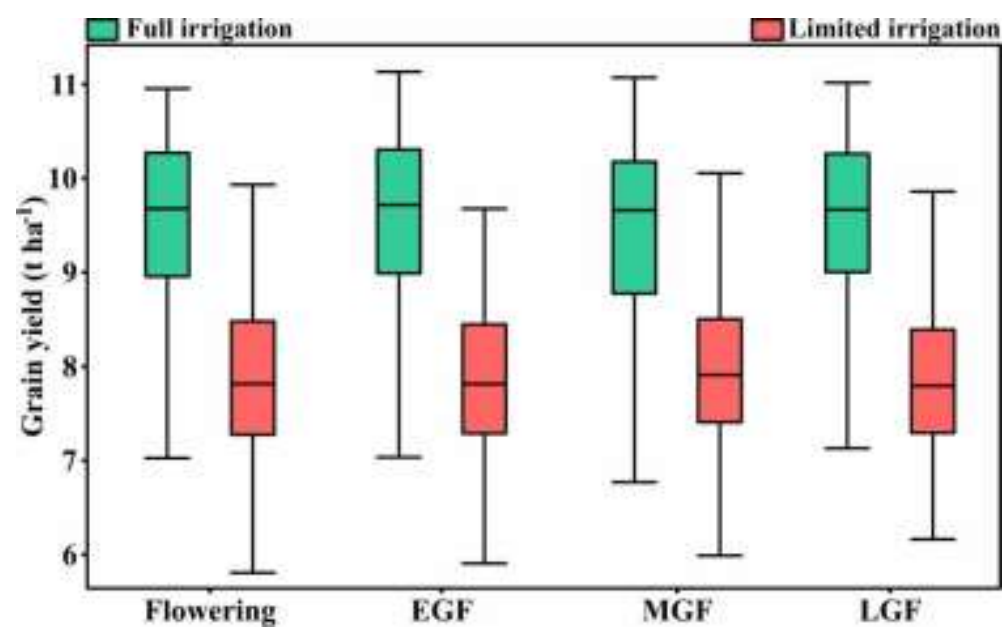

419 Fig. 10 Distribution of yield prediction values output by proposed 420 ensemble method.

421 EGF, early grain fill; MGF, min grain fill; LGF, late grain fill.

422 Table 1 Analysis of variance for predicted grain yield output by ensemble 423 feature selection method and measured grain yield.

\begin{tabular}{lllll}
\hline \multirow{2}{*}{ Grain yield } & F-value & & \\
\cline { 2 - 4 } & Genotype $(\mathrm{G})$ & Treatment $(\mathrm{T})$ & $\mathrm{G} \times \mathrm{T}$ interaction & \\
\hline Flowering & $5.27 * * *$ & $1789.08 * * *$ & $1.59 * * *$ & 0.73 \\
EGF & $5.43 * * *$ & $1487.70^{2} * *$ & $1.80 * * *$ & 0.71 \\
MGF & $6.60 * * *$ & $2334.92 * * *$ & $1.68 * * *$ & 0.77 \\
LGF & $4.64 * * *$ & $2050.94 * * *$ & $2.23 * * *$ & 0.62 \\
Measured & $3.43 * * *$ & $781.97 * * *$ & $1.44 * * *$ & 0.63 \\
\hline
\end{tabular}

424 EGF, early grain fill; MGF, min grain fill; LGF, late grain fill.

\section{Discussion}

The application of canopy hyperspectral data to predict crop yields

427 in precision agriculture management is not new $[3,14,17]$. The similarity 428 of traits among a large number of breeding lines will lead to a heavy 429 workload and make it difficult to perform accurate monitoring efforts 430 [41]. The traditional method of collecting phenotypes reduces the 431 efficiency of selecting superior breeding varieties [11]. Hyperspectral 432 remote sensing method with high spectral resolution can obtain 
continuous and fine spectral profiles of terrestrial objects at wide range wavelengths [42]. In contrast to RGB and multispectral data, hyperspectral data contains a wealth of information related to plant growth and can help detect minor differences between various breeding varieties [11].

Previous studies have shown that the full bands of hyperspectral reflectance achieved higher yield prediction accuracy than the VIs set [8, 14], but the ultra-high dimensionality of the full bands makes the program run much longer. Strong collinearity and information overlap among a large number of VIs composed of hyperspectral data, thus choosing the appropriate input features thus play an important role in reducing the dimensionality and improving the prediction accuracy. For this reason, four FS methods, and a newly proposed ensemble method were applied. The results showed that the MDI method performed better among four FS methods at all growth stages, which is consistent with previous study [16] MDI score is computed based on node impurity, which is a measure of homogeneity of the variable [16]. Our selected vegetation indices used a wide band interval, which makes a lot of vegetation indices vary widely from each other. In addition, since MDI is a tree-based scoring measure, the problem of multicollinearity was avoided, the features selected are simple and efficient [16]. Compared to MDI, only a few have investigated the effectiveness of Boruta in estimating crop parameters [11, 43]. The Boruta method in this study showed a predictive performance second only to MDI (Flowering, EGF and MGF) or comparable to MDI (LGF). The excellent performance of MDI and Boruta methods proved the usefulness of random forest algorithms in selecting features. The Fealect method has only been used in the diagnosis of lymphoma and has 
shown satisfactory classification results [36], and this study is the first to deal with the regression problem. FeaLect showed better prediction results than Relief and full features and could be widely used to investigate its potential in estimating crop parameters. The Relief method performs the worst, this is probably a result that Relief was initially designed to deal with binary classification problems and has been applied to select features in classification problems in most subsequent studies [44-46]. The advantage of the Relief method is that it does not require training, which helps to save program running time. As a deep learning method, DNN has shown high prediction accuracy in assessing crop yields and has advantages in handling large samples of complex nonlinear data [23]. Although DNNs are generally considered to be good at extracting information from raw features, this study suggests that feature engineering in deep learning is still beneficial. The feature selection in deep learning has also been performed by previous report when combining remote sensing data and DNN to predict crop yield [24].

Different FS methods may yield feature subsets that can be considered as local optima in the feature subset space, while ensemble FS can combine the local optima in each feature subset space to obtain a better model performance. The ensemble FS method proposed by [47] selected the top-ranked features of multiple FS methods but ignored the heterogeneity of each FS method and the diversity of features. A new ensemble FS method based on the stacking regression was proposed in this study. The results showed that the ensemble method improved prediction accuracy at all growth stages and gave good predictions of wheat yield, and the significant differences between treatments and varieties indicated the practical value of the proposed ensemble method in 
screening target varieties (Table 1). Overall, the newly proposed ensemble method maximizes the potential of the huge VIs dataset for crop yield predicting, allowing the best yield trait-related information to 490 be fully utilized. Meanwhile, the unsatisfactory performance of Relief inspires future research to try to combine more model training-based FS methods, such as Recursive Feature Elimination (RFE) and Variable Importance in Projection (VIP), to explore a more reasonable combination of multi-FS methods. To verify the adaptability of the proposed ensemble method, the experiments will be conducted on other crops for future analyses.

Winter wheat canopy display different structural properties during the growth cycle, which affects the optical signal at different growth stages [48]. Therefore, the difference in collection time may lead to some prediction errors of winter wheat yield. The middle and late stages of wheat growth were proven to have higher yield prediction accuracy compared to the early stages $[9,19]$. Our results indicate that the MGF achieved the highest prediction accuracy, which can help to save the number of data collection and reduce the cost.

\section{Conclusions} optimize field management practices. The use of remote sensing platforms to predict yield has been widely applied, which is not only a fast way to collect data but also reduces labor costs and problems associated with destructive sampling. In our study, an ensemble method that combines multiple FS methods based on deep neural network (DNN) and hyperspectral vegetation indices was developed. The results indicated that mean decrease impurity (MDI) performs best in grain yield 
prediction among the four FS methods at most measured stages, followed

515 by Boruta, FeaLect, and Relief. The ensemble method outperformed all

516 individual FS methods, and the highest accuracy was achieved at the mid

517 grain filling (MGF) stage. Our study demonstrated the efficacy of using

518 hyperspectral VIs and the proposed ensemble method for predicting

519 wheat yield. For future work, comprehensive studies in different

520 environments are still needed to validate the transferability of this

521 ensemble method and to identify the best combination of FS methods.

522 Ethics approval and consent to participate

523 Not applicable.

524 Consent for publication

525 All authors agreed to publish this manuscript.

526 Availability of data and materials

527 All the data used in this study is available upon the approval of Dr.

528 Yonggui XIAO from the Institute of Crop Sciences, Chinese Academy of

529 Agricultural Sciences, China.

530 Competing interests

531 The authors declare that they have no known competing financial

532 interests or personal relationships that could have appeared to influence

533 the work reported in this paper.

$534 \quad$ Funding

535 This work was funded by the National Natural Science Foundation of

536 China (31671691).

537 Authors' contributions

538 S.F. and L.L. collected the data, S.F. analyzed the data and wrote the 539 paper, Z.C. and Y.X. managed and directed the trial, Y.X. and Z.H. gave 540 comments and suggestions to improve the manuscript. All authors have 
read and agreed to the published version of the manuscript.

\section{Acknowledgments}

We are grateful to the authors of the open-source R packages used in this paper.

\section{References}

[1] Lesk C, Rowhani P, Ramankutty N. Influence of extreme weather disasters on global crop production. Nature. 2016;529:84.

[2] Shafiee S, Lied LM, Burud I, Dieseth JA, Alsheikh M, Lillemo M. Sequential forward selection and support vector regression in comparison to LASSO regression for spring wheat yield prediction based on UAV imagery. Comput Electron Agr. 2021;183:106036.

[3] Yoosefzadeh-Najafabadi M, Earl HJ, Tulpan D, Sulik J, Eskandari M. Application of machine learning algorithms in plant breeding: Predicting yield from hyperspectral reflectance in soybean. Front Plant Sci. 2021;11:624273.

[4] Araus JL and Cairns JE. Field high-throughput phenotyping: the new crop breeding frontier. Trends Plant Sci. 2014;19:52-61.

[5] Xiong Q, Tang G, Zhong L, He H and Chen X. Response to nitrogen deficiency and compensation on physiological characteristics yield formation and nitrogen utilization of rice Front Plant Sci. 2018;9:1075.

[6] Rutkoski J, Poland J, Mondal S, Autrique E, Gonzalez Perez L, Crossa J, et al. Canopy temperature and vegetation indices from high-throughput phenotyping improve accuracy of pedigree and genomic selection for grain yield in wheat G3 (Bethesda). 2016;6(9):2799-2808. 
[7] Luis Araus J, Cairns JE. Field high-throughput phenotyping: the new crop breeding frontier. Trends Plant Sci. 2014;19:52-61

[8] Montesinos-Lopez OA, Montesinos-Lopez A, Crossa J, Campos GDL, Alvarado G, Mondal S, et al. Predicting grain yield using canopy hyperspectral reflectance in wheat breeding data. Plant Methods. 2017;13:4.

[9] Hassan MA, Yang M, Rasheed A, Yang G, Reynolds M, Xia X, et al. A rapid monitoring of NDVI across the wheat growth cycle for grain yield prediction using a multi-spectral UAV platform Plant Sci. 2019;282:95-103.

[10] Messina G, Modica G. Applications of UAV thermal imagery in precision agriculture: State of the art and future research outlook Remote Sens. 2020;12(9):1491.

[11] Shu M, Zuo J, Shen M, Yin P, Wang M, Yang X, et al. Improving the estimation accuracy of SPAD values for maize leaves by removing UAV hyperspectral image backgrounds. Int J Remote Sens. 2021;42:5862-5881.

[12] Yamaguchi T, Tanaka Y, Imachi Y, Yamashita M, Katsura K. Feasibility of combining deep learning and RGB images obtained by unmanned aerial vehicle for leaf area index estimation in rice. Remote Sens. 2021;13(1):84.

[13] Huang Z, Liu F, Hu G. The novel method for LAI inversion using Lidar and hyperspectral data Funct Mater. 2017;24:442-450.

[14] Li B, Xu X, Zhang L, Han J, Bian C, Li G, et al. Above-ground biomass estimation and yield prediction in potato by using 
UAV-based RGB and hyperspectral imaging. ISPRS J Photogramm Remote Sens. 2020;162:161-172.

[15] Shah SH, Angel Y, Houborg R, Ali S, McCabe MF. A random forest machine learning approach for the retrieval of leaf chlorophyll content in wheat. Remote Sens. 2019;11:920.

[16] Bhadra S, Sagan V, Maimaitijiang M, Maimaitiyiming M, Newcomb M, Shakoor N, et al. Quantifying leaf chlorophyll concentration of sorghum from hyperspectral data using derivative calculus and machine learning. Remote Sens. 2020;12:2082.

[17] Feng L, Zhang Z, Ma Y, Du Q, Williams P, Drewry J, et al. Alfalfa yield prediction using UAV-Based hyperspectral imagery and ensemble learning. Remote. Sens. 2020;12:2028.

[18] Lopez-Cruz M, Olson E, Rovere G, Crossa J, Dreisigacker S, Mondal S, et al. Regularized selection indices for breeding value prediction using hyper-spectral image data. Sci Rep. 2020;10:1-12.

[19] Fei S, Hassan MA, He Z, Chen Z, Shu M, Wang J, et al. Assessment of Ensemble Learning to Predict Wheat Grain Yield Based on UAV-Multispectral Reflectance. Remote Sens. 2021;13: 2338

[20] Maimaitijiang M, Ghulam A, Sidike P, Hartling S, Maimaitiyiming M, Peterson K, et al. Unmanned Aerial System (UAS)-based phenotyping of soybean using multi-sensor data fusion and extreme learning machine. ISPRS J Photogramm Remote Sens. 2017;134:4358.

[21] Wang L, Zhou X, Zhu X, Dong Z, Guo W. Estimation of biomass in wheat using random forest regression algorithm and remote sensing 
data. Crop J. 2016;4:212-219.

[22] Maimaitijiang M, Sagan V, Sidike P, Hartling S, Esposito F, Fritschi FB. Soybean yield prediction from UAV using multimodal data fusion and deep learning Remote Sens Environ. 2020;237:111599.

[23] Jin X, Li Z, Feng H, Ren Z, Li S. Deep neural network algorithm for estimating maize biomass based on simulated Sentinel 2A vegetation indices and leaf area index. Crop J. 2020;8:87-97.

[24] Sagan V, Maimaitijiang M, Bhadra S, Maimaitiyiming M, Brown DR, Sidike P, et al. Field-scale crop yield prediction using multi-temporal WorldView-3 and PlanetScope satellite data and deep learning. ISPRS J Photogramm Remote Sens. 2021:174:265-281.

[25] Ishengoma FS, Rai IA, Said RN. Identification of maize leaves infected by fall armyworms using UAV-based imagery and convolutional neural networks. Comput Electron Agr. 2021;184 (12):106124.

[26] Hennessy A, Clarke K and Lewis M. Hyperspectral classification of plants: a review of waveband selection generalisability. Remote Sens.2020;12:113.

[27] Fu P, Meacham-Hensold K, Guan K, Bernacchi CJ. Hyperspectral leaf reflectance as proxy for photosynthetic capacities: An ensemble approach based on multiple machine learning algorithms. Front Plant Sci. 2019;10:624273.

[28] Wang L, Zhu Z, Sassoubre L, Yu G, Liao C, Hu Q, Wang Y. Improving the robustness of beach water quality modeling using an 
ensemble machine learning approach. Sci Total Environ. 2021;765: 142760.

[29] Frame J, Merrilees DW. The effect of tractor wheel passes on herbage production from diploid and tetraploid ryegrass swards. Grass Forage Sci. 1996;51:13-20.

[30] Chen K, Chen K, Wang Q, He Z, Hu J, He J. Short-Term load forecasting with deep residual networks. IEEE $\mathrm{T}$ Smart Grid. 2019;10:3943-3952.

[31] Huete A, Didan K, MiuHu Q, Zhang R and Zhou Y. Transfer learning for short-term wind speed prediction with deep neural networks. Renewable Energy. 2016;85:83-95.

[32] Montesinos-Lopez OA, Martin-Vallejo J, Crossa J, Gianola D, Hernandez-Suarez CM, Montesinos-Lopez A, Juliana P, Singh R. A benchmarking between deep learning support vector machine and bayesian threshold best linear unbiased prediction for predicting ordinal traits in plant breeding. G3 Genes Genomes Genet. 2019;9: 601-618.

[33] Breiman L. Random forests. Mach Learn. 2001; 45:5-32.

[34] Stoppiglia H, Dreyfus G, Dubois R, Oussar Y. Ranking a random feature for variable and feature selection. J Mach Learn Res. 2003;3 (Mar):1399-1414.

[35] Masrur Ahmed AA, Deo RC, Feng Q, Ghahramani A, Raj N, Yin Z, et al. Deep learning hybrid model with Boruta-Random forest optimiser algorithm for streamflow forecasting with climate mode indices rainfall and periodicity J Hydrol. 2021;599:126350. 
667

668

669

670

671

672

673

674

675

676

677

678

679

680

681

682

683

684

685

686

687

688

689

690

691

[36] Zare H, Haffari G, Gupta A, Brinkman RR. Scoring relevancy of features based on combinatorial analysis of Lasso with application to lymphoma diagnosis. BMC Genomics. 2013;14 Suppl 1 S14.

[37] Park H, Kwon H. Extended relief algorithms in instance-based feature filtering. International Conference on Advanced Language Processing \& Web Information Technology. IEEE. 2007, pp 123128.

[38] Robnik-Šikonja M, Kononenko I. Theoretical and empirical analysis of ReliefF and RReliefF Mach Learn 2003;53:23-69.

[39] Wu T, Zhang W, Jiao X, Guo W, Alhaj Hamoud Y. Evaluation of stacking and blending ensemble learning methods for estimating daily reference evapotranspiration. Comput Electron Agr. 2021;184: 106039.

[40] Hassan M, Yang M, Rasheed A, Jin X, Xia X, Xiao Y, et al. Time-series multispectral indices from unmanned aerial vehicle imagery reveal senescence rate in bread wheat. Remote Sens. 2018; 10:809.

[41] Duan T, Chapman SC, Guo Y, Zheng B. Dynamic monitoring of NDVI in wheat agronomy and breeding trials using an unmanned aerial vehicle. Field Crop Res. 2017;210:71-80.

[42] Fan L, Zhao J, Xu X, Liang D, Yang G, Feng H, et al. Hyperspectral-based estimation of leaf nitrogen content in corn using optimal selection of multiple spectral variables Sensors. 2019;19(13):2898.

[43] Tatsumi K, Igarashi N, Mengxue X. Prediction of plant-level tomato 
biomass and yield using machine learning with unmanned aerial vehicle imagery. Plant Methods. 2021;17:77.

[44] Karami K, Akbari M, Moradi M, Soleymani B, Fallahi H. Survival prognostic factors in patients with acute myeloid leukemia using machine learning techniques. Plos One. 2021;16(7):e0254976.

[45] Kira K, Rendell LA. A practical approach to feature selection proceedings of the ninth international workshop on machine learning. Aberdeen Scotland UK July 1-3 1992.

[46] Maurya NS, Kushwaha S, Chawade A, Mani A. Transcriptome profiling by combined machine learning and statistical $\mathrm{R}$ analysis identifies TMEM236 as a potential novel diagnostic biomarker for colorectal cancer. Sci Rep. 2021;11:14304

[47] Saeys YT, Abeel and Y Peer. Robust feature selection using ensemble feature selection techniques. Springer Berlin Heidelberg Berlin Heidelberg. 2008; pp 313-325.

[48] Jin X, Li Z, Yang G, Yang H, Feng H, Xu X, et al. Winter wheat yield estimation based on multi-source medium resolution optical and radar imaging data and the AquaCrop model using the particle swarm optimization algorithm. ISPRS J Photogramm Remote Sens. 2017;126:24-37. 


\section{Supplementary Files}

This is a list of supplementary files associated with this preprint. Click to download.

- Appendix.docx 